\title{
Measuring the Magnetic Moments of Short-Lived Particles Using Channeling in Bent Crystals
}

V.V. Baublis, A.V. Khanzadeev, V.M. Samsonov, N.K. Terentyev and A.A. Vorobyov Petersburg Nuclear Physics Institute, Gatchina, Russia

R.A. Carrigan, Jr., D. Chen, P.S. Cooper, D.C. Daniels, J. Lach and A. Morelos

Fermi National Accelerator Laboratory, P.O. Box 500, Batavia, Illinois 60510

V.J. Smith

H.H. Wills Physics Laboratory, University of Bristol, United Kingdom

C.R. Sun

State University of New York at Albany, Albany, New York 12222

S. Timm

Carnegie-Mellon University, Pittsburgh, Pennsylvania 15213

M.N. Strikhanov

Moscow Engineering Phyiscs Institute, Moscow 115409. Russia

March 1994

Accepted in Nuclear Instruments and Methods 


\section{Disclaimer}

This report was prepared as an account of work sponsored by an agency of the United States Government. Neither the United States Government nor any agency thereof, nor any of their employees, makes any warranty, express or implied, or assumes any legal liability or responsibility for the accuracy, completeness, or usefulness of any information, apparatus, product, or process disclosed, or represents that its use would not infringe privately owned rights. Reference herein to any specific commercial product, process, or service by trade name, trademark, manufacturer, or otherwise, does not necessarily constitute or imply its endorsement, recommendation, or favoring by the United States Government or any agency thereof. The views and opinions of authors expressed herein do not necessarily state or reflect those of the United States Government or any agency thereof. 
MEASURING THE MAGNETIC MOMENTS OF SHORT-LIVED PARTICLES USING CHANNELING IN BENT CRYSTALS

V. V. Baublis(1), R. A. Carrigan Jr. (2), D. Chen (2) P. S. Cooper ${ }^{(2)}, D^{\prime} C_{j}$ Daniels ${ }^{(2) a}$, A. V 'Khanzadeev ${ }^{(1)}$ J. $\operatorname{Lach}^{(2)}$, A. Morelos (2) , V. M. Samsonov (i), V. J. Smith'(3), M. N. Strikhanov $(6), C^{\prime}$. R. Sun $(4), N$. K. 'Terentyev ${ }^{(1)}$, S. Timm ${ }^{\prime}$, A. A. Vorobyov $(1)$

(1) Petersburg Nuclear Physics Institute, Gatchina, Russia

(2) Fermi National Accelerator Laboratory, Batavia, IL 60510, USA (3) H.H. Wills Physics Laboratory, University of Bristol, UK (4) State University of New York-Albany, Albany, NY 12222, USA (5) Carnegie-Mellon University, Pittsburgh, PA 15213, USA (6) Moscow Engineering Physics Institute, Moscow, 115409, Russia

\section{ABSTRACT}

A recent demonstration of spin precession using channeling in a bent crystal offers the interesting possibility of a technique to measure magnetic moments of short-lived charm baryons. There are significant differences among theoretical models of these moments. Accumulating experimental evidence indicates the tools used for hyperon magnetic moment measurements, polarized production and decay asymmetries, may also be available with heavier baryons. Useful measurements may be possible using the channeling technique but they will require challenging beam conditions. 
Introduction

The spin of a channeled particle ${ }^{1}$ moving in a bent crystal should precess through an angle $\phi$ given by:

$$
\phi=\gamma \omega(g-2) / 2
$$

for $\gamma \gg 1$, where $\gamma$ is the Lorentz factor, $g$ is the gyromagnetic ratio, and $\omega$ is the deflection angle of the channeled particle ${ }^{2}$. In a recent experiment at Fermilab ${ }^{3}$ this spin precession was observed for the first time. Polarized $\Sigma^{+}$from the Fermilab charged hyperon beam were channeled in two $4.5 \mathrm{~cm}$ long silicon crystals with 1.65 mrad bends, resulting in a spin precession of $60 \pm 17^{\circ}$. This was in agreement with the predicted value of $62^{\circ}$ based on the world average of the measurements of the $\Sigma^{+}$magnetic moment. During the precession the magnitude of the $\Sigma^{+}$polarization remained unchanged to within the precision of the measurement.

The spin precession can be ascribed to an equivalent magnetic field giving rise to the same angular deflection as the bent crystal. This is more than a metaphor since the electric fields that channel the particles Lorentz transform into the equivalent magnetic field. The equivalent magnetic field for bent crystal channeling for the case $\beta \approx 1$ is:

$$
\mathrm{B}=\mathrm{p} / 0.3 \mathrm{R}
$$

(here $B$ is in tesla, $p$, the momentum, is in $\mathrm{GeV} / \mathrm{c}$, and $R$, the radius of curvature, is in $\mathrm{m}$ ). The minimum value of the crystal radius of curvature is the Tsyganov radius ${ }^{4}$, the radius where the particle will no longer channel since the centrifugal force overcomes the channeling. In the extreme relativistic limit the Tsyganov radius is:

$$
\mathrm{R}_{\mathrm{T}}=\mathrm{pc} / \mathrm{eE}_{\mathrm{C}}
$$

where $e$ is the charge of the electron and $E_{C}$ is the critical field at which the particle no longer channels. In practical experiments the radius of curvature must be several times larger than the Tsyganov radius to avoid significant dechanneling. The radius of curvature in the recent Fermilab experiment using a silicon crystal was $27 \mathrm{~m}$ or ten times the Tsyganov radius. The Tsyganov radius is proportional to $1 / \mathrm{z}$ where $\mathrm{z}$ is the nuclear charge of the crystal material. Increasing from $\mathrm{Z}=14$ (Si) to $\mathrm{Z}=32$ (Ge) or $\mathrm{Z}=74(\mathrm{~W})$ could give substantially higher fields as well as larger channeling critical angles. (The critical angle determines the angular acceptance for channeled particles.) Note, however, that crystals with low dislocation densities are needed for high energy channeling. It is here that $\mathrm{Si}$ excels. For the Fermilab demonstration the equivalent magnetic field was 45 tesla. Channeling in single crystals could be preserved for equivalent fields to order $1000 \mathrm{~T}$. 
Because they produce large deflections in a short length of crystal, these high effective magnetic fields associated with bent crystal channeling offer a unique possibility for the measurement of the magnetic moments of particles with lifetimes such that their mean path lengths are order $1 \mathrm{~cm}$. These path lengths are characteristic of a charm baryon with energy in the several hundred GeV range. On the other hand, the angular acceptance for planar channeling is small, on the order of 10 urad for silicon at 400 GeV. This should be compared to typical particle production distributions which are in the 1 mrad range. The channeling angular acceptance represents a significant limitation for applying channeling to magnetic moment measurements.

This note first examines potential improvements to the Fermilab channeling experiment as well as other recent developments, then considers their application to measuring charm baryon magnetic moments. To apply the channeling technique to charm baryon magnetic moment measurements it is first necessary to understand whether the parameters of the recent Fermilab test can be scaled to more ambitious experiments. Next it is necessary that the short-lived particles be produced with some polarization and that decay modes exist with non-zero values of $\alpha$ (the asymmetry parameter ${ }^{5}$ ) to measure the polarization precession. Finally, it is essential to accumulate a large enough sample of decays to do a precession analysis. Since the error on the asymmetry $\Delta A=\sqrt{3 / N}$, a 108 measurement for a typical polarization requires on the order of $\mathbf{N}=10,000$ events.

An important question is whether such measurements are useful. Recent theoretical results bearing on this point are also discussed.

Could the Fermilab Measurement of the $\Sigma^{+}$Hyperon Magnetic Moment Be Improved?

To answer this question it is useful to look at the event flow for the recent Fermilab experiment. Table 1 gives a detailed presentation of the predicted number of events based on the flux and the expected channeling properties. These results and the uncertainties are summarized in the thesis of Chen ${ }^{6}$. This table recapitulates the event flow from the total number of particles through the system to the final sample. It is broken down into several parts: 1) the total flux of particles in the experiment, 2) the number of $\Sigma$ that decayed within the active volume after the channeling crystal and were accepted by the system, 3) the channeling acceptance including spatial and angular effects as well as dechanneling, and 4) the number that pass through the analysis cuts. The predicted and observed number of events agree to within a factor of two, which is consistent with the uncertainties of the comparison. 
A second approach that is more experimentally-based has also been used to check the transmission. This is done by comparing the observed number of long-lived channeled particles to the expected number for one run. This eliminates problems related to summing alternating polarizations over many runs, uncertainty as to the fraction of $\Sigma$ in the beam, and the $\Sigma$ decay volume. In his thesis Chen finds that the theoretical rate is 50\% larger than the experimental rate and that the uncertainty is $50 \%$ so that this comparison is also consistent.

Some of the factors where there are opportunities for improvement have been listed in Table 2. Different choices of the beam energy and $x_{F}$ (Feynman $x$ ) for the $\Sigma$ in other high energy experiments have produced polarizations up to $P=0.2$ but at some cost in rate. For E761, running at a small $p_{t}$ would have produced a larger polarization and a higher rate. By decreasing the radius of curvature by a factor of 3 to 5 and lengthening the crystal it is possible to achieve bending angles using channeling that are comparable to magnetic deflections used in earlier precision experiments. The spatial acceptance could be increased by a factor of twenty-five by increasing the active region of the crystals and making them slightly thicker as well as by using a second set of crystals. Of course it is necessary to be sure the phase space of the crystals is matched to the phase space of the beam. With these modifications it would take about 60 hours of running to match the statistics of the precision experiments ${ }^{7}$ carried out in the eighties.

The systematic uncertainties associated with the bent crystal technique and magnetic deflection are not that different. The biggest intrinsic uncertainty in the channeling experiment is associated with the channeling deflection angle. For the recent test the uncertainty in the deflection angle was $25 \mu \mathrm{rad}$. For a 10 mrad deflection this would result in a $0.25 \%$ systematic uncertainty.

While these comparisons are interesting, channeling appears to have no role for future precision measurements of conventional hyperons. The E761 experiment where the channeling test was carried out also accumulated ten times the sample of the previously-published $\Sigma^{+}$magnetic moment experiments for a magnetic moment measurement. Thus it would appear that the channeling technique would always be at a disadvantage because of the limited acceptance. Bent crystal channeling holds no advantage for hyperon magnetic moment measurements because normal magnetic fields are strong enough to precess hyperons a sufficient amount at high energy before they decay. 
Theoretical Predictions of Charm Baryon Magnetic Moments:

Theoretical modeling of hyperon and other baryon magnetic moments has a long, interesting, and not entirely successful history. The simple non-relativistic quark model ${ }^{8}$ explained the ratio of the neutron and proton magnetic moments. SU(3) ${ }^{9}$ (for example the independent quark model of De Rujula-Georgi-Glashow ${ }^{10}$ ) related the $\Lambda$ moment to the $\Sigma$. Currently none of the magnetic moment models successfully predict all the features of the hyperon moments. Some are unconcerned by this disagreement, arguing that the details could be determined with a full lattice gauge theory calculation and that little new would be learned at this time by modeling the experimental data. However others suggest that these discrepancies can yield valuable insights into the non-perturbative structure of hadrons.

The present experimental information only partially covers the baryon magnetic moments. A reasonable question is whether it would be worthwhile to measure some of the remaining ones. One answer is that a charm baryon magnetic moment measurement would give some insight into the magnetic moment of the charm quark. Specifically, in the simple quark model the magnetic moment of the $\Lambda_{c}$ baryon is the magnetic moment of the charm quark. This is exactly the same as the case for the $\Lambda^{0}$ where the other two light quarks are both members of the same isospin multiplet in a spin singlet state and the $I=0$ heavy quark (the strange quark in the $\Lambda^{0}$ case) is required to have zero orbital angular momentum. The magnetic moment of the charm quark should be related to the strange quark as the ratio $2 \mathrm{~m}_{\mathrm{s}} / \mathrm{m}_{\mathrm{C}}$. Azimov ${ }^{11}$ notes that confirmation of the simple quark model prediction for the $\Lambda_{c}$ moment would be evidence supporting the existence of a cloud of light quarks around a heavy constituent quark.

A number of theoretical groups have made estimates of the charm baryon magnetic moments. An early calculation by Choudhury and Joshi12 used the $U(4)$ symmetry group, while Lichtenberg ${ }^{13}$ employed SU(4). Pandit et al. ${ }^{14}$ employed a variation on the independent quark model along the lines of De Rujula-Georgi-Glashow to calculate the moments. Jena et al. ${ }^{15}$ also used the independent quark model with a logarithmic potential in the spirit of the Quigg-Rosner ${ }^{16}$ calculations of the charm mass splittings. Bose and Singh ${ }^{17}$ based their estimates on the MIT bag model for the potential while oh et al. ${ }^{18}$ employed a topological soliton model following the work of Riska et al.19. Surprisingly, this model agrees quite closely with quark model predictions in many cases. Table 3 lists several of these predictions for positively-charged baryons with one unit of charm that might be able to be measured with bent crystal channeling. In the simplest models, the $\Xi^{+}$has the same magnetic moment as the $\Lambda^{+}{ }_{c}^{\prime}$ but one might expect to see mixing with the $\Xi^{+} c^{\prime}$, producing a corresponding change in the magnetic moment of the physical $\Xi^{+}{ }_{c}$ state. 
As in the case of the hyperon magnetic moments, there are significant differences between the models. For example, estimates for the $\Xi^{+}$magnetic moment differ by a factor of two. Several of these calculations are more than a decade old so some of the differences are due to the use of different information for potentials and charm mass. It would be useful to understand the differences between the models in more detail. It does appear that there should be interesting physics that would be illuminated by measurements of charm magnetic moments.

Extending Channeling Magnetic Moment Measurements to Charm Baryons

In the introduction it was pointed out that crystal channeling is quite well suited to magnetic moment measurements of short-lived particles from the standpoint of achieving substantial spin precession within the particle lifetime. For example, while the decay length of a $300 \mathrm{GeV} / \mathrm{c} \Sigma^{+}$is $6 \mathrm{~m}$, the decay length for a $\Lambda_{c}$ with the same momentum is $7.5 \mathrm{~mm}$, so the high effective field of a crystal is needed to achieve some precession. As noted earlier, three other elements are needed to carry out a spin precession measurement: 1) a production mechanism that produces polarized, short-lived baryons, 2) a decay mode with a non-zero asymmetry parameter to use as a polarization analyzer, and 3) samples of channeled, polarized short-lived baryons on the order of 10,000 events or more.

A group working with the BIS-2 spectrometer at Serpukhov 20 has reported a limit on the polarization of $\Lambda_{c}$ produced by $40-70$ $\mathrm{GeV}$ neutrons that is about two $\sigma$ away from zero and in line with hyperon polarizations. More recently Jezabek et al.21 have reported evidence for polarization in a 121 event sample from the ACCMOR detector at CERN (NA-32). They look at the decay mode $\Lambda^{+}{ }_{c} \rightarrow \mathrm{pk}^{-} \pi^{+}$, a mode with a branching ratio of $3.2 \%$. Because this is a three-body decay they must use a technique similar to the approaches suggested by Berman and Jacob ${ }^{22}$ or Bjorken ${ }^{23}$. The Bjorken approach extends the alpha, beta, gamma formalism used for two-body hyperon decays to multi-body modes. Note that information on the final charm baryon spin direction could also be inferred from observation of the decay of the daughter baryon. For example, the $\Lambda^{0}$ will carry information on the spin direction of its parent.

Groups at Cornel1 $1^{24}$ and $\mathrm{DESY}^{25}$ have now reported measurements of the $\Lambda_{C}$ decay asymmetry parameter for the decay $\Lambda_{C} \rightarrow \Lambda \pi^{+}$. Both groups get values of $\alpha$ that are about $-1.0 \pm 0.4$ so that the asymmetry is pleasingly large. This is also in line with theoretical estimates. The branching ratio to this channel is about 0.68 . Small branching ratios to two body states are a general problem for charm baryon studies. On the other hand, the Bjorken formalism may be able to be exploited for the other states. 
Unfortunately one other element is needed, the sample of ten thousand or so channeled, polarized charm baryons. The largest sample of $\Lambda_{C} \rightarrow$ pK $\pi$ so far published for fixed target running in a hadron beam is 154 events by ACCMOR in 199026, the data set used for the Jezabek et al. analysis. Currently the published world sample of $\Lambda_{c}$ is less than 2000. This is a long way from what is required. E791, a high-statistics charm production experiment at Fermilab, now has an order of magnitude more data on tape than the ACCMOR sample. (However there may be little or no polarization since the baryons are produced by pions at small angles.) E781, an experiment to study charm baryon production at Fermilab, expects to get samples of 70,000 events per decay channel. E781 will be carried out during the next Fermilab fixed target running period.

There are also two further technical complications related to channeling that must be considered. The first is that useful bentcrystal channeling only occurs for positive particles. This rules out measurements of the magnetic moments of negative particles. The second complication is that it will not be possible to use the channeling crystal itself as a target for short-lived particle production. That is because the nuclei in the crystal are at the edges of the channels so that it is not possible to capture a charm particle produced in a crystal into a channel. This means that a separate target must be used upstream of the channeling crystal. The length of the target could not be much longer than the charm particle mean path length. This complication might be balanced by the fact that the channeled short-lived particles are bent out of the beam so that it may be possible to build an effective, highrate trigger. An advantage of charm baryon production with a negative hyperon beam is that the beam particles would not channel.

\section{Potential Magnetic Moment Measurements}

Studies of potential charm magnetic moment measurements are needed for several reasons. One is to understand the beam rate required to do a useful measurement in a reasonable time. A second is to gain some insight into the key parameters that would enter into a measurement such as the momentum and angular distribution of the charm baryons and their decay products. Knowledge of these parameters will help in the design of triggers that can work at the high beam rates that are expected to be required.

We have used a Monte Carlo study coupled with an analytical calculation to investigate rate questions as well as the effects of crystal length and bend angle. The study was done in the context of Fermilab E781. For the study, particles were assumed to be produced by a $600 \mathrm{GeV} / \mathrm{c}$ beam. (E781 will operate at $650 \mathrm{GeV} / \mathrm{C}$.) Amorphous tungsten was used as the target to get high nuclear density. Two different production processes were considered. A proton beam was assumed for $\Lambda_{c}$ production while for the $\Xi_{c}$ case a hyperon beam was employed to enhance polarization and charm 
production in the spirit of the proposed E781 experiment. Conventional parameterizations 27 of $x_{F}$ and $p_{t}$ were used for the charm production cross sections.

Polarization was assumed to be 0.2. This may be optimistic. The asymmetry parameter was taken to be $\alpha=1$, in line with the recent CLEO and ARGUS measurements for $\Lambda_{c} \rightarrow \Lambda \pi^{+}$. The study used the channeling parameters from the Fermilab E761 test, that is, a Si crystal bent along the (111) plane to deflect the charm baryon and precess its spin. The front face of the crystal was assumed to be large enough to accept all of the incident production flux. This appears to be reasonably easy to achieve. No detector efficiencies were folded into the calculation.

The number of channeled charm baryons produced is a product of eight factors:

1. the probability that the production particle reacts one and only one time in the target,

2. the fraction of the interactions that produce charm baryons that decay to the mode under study, and

3. the probability of the charm particle not decaying before it exits after channeling through the full length of the deflecting crystal. This depends on the momentum of the particle.

4. The channeling acceptance. This is proportional to the critical angle and related to the production transverse momentum folded with the beam angular distribution and

5. $x_{F}$.

5. The surface acceptance for channeling. This is due to the microstructure of the lattice and is assumed to be $1 / 2$.

6. The likelihood that the particle does not undergo ordinary dechanneling and

7. the likelihood that the particle does not undergo bending dechanneling. This decreases as the radius of curvature of the crystal decreases and depends on the momentum.

8. The requirement that $p_{t}$ be large enough so that the particle is polarized.

These eight terms lead to an expression that was evaluated using a Monte Carlo for different values of the target and crystal length as well as the crystal bend angle with the requirement that there were enough events for a $10 \%$ measurement of the magnetic moment.

The angle and crystal length given by the calculation are large and lead to a rather large charm baryon attenuation. For the optimization for the $\Lambda_{c}$ the tungsten target thickness is $0.6 \mathrm{~cm}$, the crystal length is $2 \mathrm{~cm}$, and the crystal bend angle is $15 \mathrm{mrad}$. The precession is -0.054 radians for a naive quark model magnetic moment. For the $\Xi_{\mathrm{c}}$ the optimum target thickness is $2.3 \mathrm{~cm}$, the crystal length is $2.2 \mathrm{~cm}$, and the bend angle is $5 \mathrm{mrad}$. The 
precession is -0.008 radians. For both the $\Lambda_{c}$ and the $\Xi_{C}$ the average momentum of the channeled particles that survive and are suitable for a spin precession measurement is about half the incident beam momentum.

A ten percent measurement of the magnetic moment of the $\Lambda^{+}$ using the decay $\Lambda_{\mathrm{C}}{ }^{+} \rightarrow \mathrm{pK}^{-} \pi^{+}$requires about 600 hours in a $10^{9}$ particles/s proton beam. Such a beam would not permit operation of normal in-beam single particle detectors. A $\Xi_{C}$ measurement using a very optimistic $10^{8} \mathbf{s}^{-1}$ hyperon beam would require about 5000 hours.

The calculation is not sensitive to the target length provided the target is about one charm baryon decay length thick. The maxima for the deflection angle and the crystal length are also relatively shallow so that a serious optimization could be made based on other factors, such as background. For example, background considerations might dictate a large bending angle while the desire for a large number of charm candidates suggests a short crystal and a smaller bend.

A technique called bias canceling has been used in most high energy hyperon magnetic moment measurements. The hyperon polarization is reversed frequently to cancel out systematic uncertainties. If the charm baryons are produced by a polarized $\Sigma$ beam and that gives spin transfer (as may be tried in Fermilab E781), a conventional bias canceling approach can be used. The polarization of the $\Sigma$ beam can be reversed in the normal way by changing the $\Sigma$ production angle and looking at forward-produced charm baryons. On the other hand, a different approach must be followed if the charm baryons are produced by unpolarized protons. Since the charm baryons are short-lived there is no "hyperon-like" beam where the production angle can be reversed to reverse the polarization and thereby do bias canceling. The polarization will have to be established on an event-by-event basis by determining the angle between the baryon and the production particle. This weighting should be facilitated by the good angular resolution of the channel, on the order of $10 \mu \mathrm{rad}$. Exact reproduction of opposite bends will not be necessary since a single crystal will accept events with the full range of production angles and thus of polarization directions. However, the use of two crystals, or changing the bend in one crystal, could provide information on systematic effects.

Going to higher energy at an accelerator like the SSC might improve magnetic moment measurements with bent crystals ${ }^{28}$. The lifetime in the laboratory frame and $\gamma$ would increase, resulting in more precession (although the precession in conventional magnetic fields would still be very small). The cross section for charm baryon production is also expected to increase significantly. The behavior of baryon polarization with increasing beam energy depends 
on the baryon ${ }^{29}$. More studies are needed to assess the potential of higher energy.

A good trigger strategy would be crucial for a successful short-lived magnetic moment measurement. A complete optimization would have to include correlation effects and take the trigger into account. Full Monte Carlo studies are an appropriate approach for these studies.

\section{Summary}

Plausible improvements in the recent Fermilab demonstration of $\Sigma^{+}$bent-crystal spin precession could measure positive hyperon magnetic moments with a precision comparable to conventional measurements of a decade ago. However, the natural regime for application of the bent crystal technique is for measurements of the short-lived charm baryon magnetic moments where the effective fields of order one thousand tesla can produce substantial spin precession. The technique is limited by the small acceptance of the channeling process, the need for charm baryon production outside the channeling crystal, and the requirement that the channeled particle have a positive charge.

Small samples of charm baryons suggest that they will exhibit the same intriguing polarizations seen with the hyperons. Recent experiments have also shown that some charm baryon two-body decays have non-zero asymmetry parameters. These two facts indicate that the current techniques for spin precession used for hyperons could be carried over to charm baryon magnetic moment measurements. It would be useful to have more information on these two areas, particularly on the polarization and the practical application of the Bjorken spin analysis.

Heroic beam rates will probably be required, in part because of the small channeling acceptance and in part because branching ratios to particular final states are small. In turn, these rates place extreme requirements on the detector instrumentation. These problems may be partially balanced out by substantial deflections of the short-lived particles that result from using the bent crystals.

While application of bent crystal channeling to short-lived magnetic moment measurements would be challenging the experiments may eventually be within reach. The possibilities should not be overlooked in planning for future charm baryon experiments. Continuing theoretical and experimental investigations of these polarization processes, asymmetries, and magnetic moments appear to be warranted.

We have not discussed the measurement of beauty baryon magnetic moments with the bent crystal technique. While beauty 
particle lifetimes are comparable to charm lifetimes and the expected magnetic moments are in the same range, the beauty production cross sections are very small at Tevatron fixed target energies. Even at LHC fixed target energies the possibility is daunting. An additional problem is that the beauty quark has a negative charge so that the beauty baryons have negative or neutral charges and could not be channeled. Positively-charged anti particles could be channeled but the production rates would be smaller yet.

We wish to thank the other members of Fermilab E-761 for their help. This work was supported in part by the U.S. Department of Energy under contracts DE-AC02-76CH0300, DE-FG02-91ER40631, DE-FG02-91ER40682, the Russian Academy of Sciences and the UK Science and Engineering Council.

a Now at Harvard University, Cambridge, MA 02138, USA.

$b$ Now at the SSC Laboratory, Dallas, TX 75237, USA. 
References:

1. See, for example, Relativistic Channeling, eds. R. A. Carrigan, Jr. and J. E. Ellison (Plenum, New York, 1987).

2. V. G. Baryshevskii, Pis'ma Zh. Tekh. Fiz. 5, 182 (1979), Sov. Tech. Phys. Lett. 5, 73 (1979).

L. Pondrom, private communication and Proc. of the 1982 DPF Summer School on Elementary Particle Physics and Future Facilities, p. 98, eds. R. Donaldson, R. Gustafson, and F. Paige, Snowmass, CO (1982).

V. L. Lyuboshits, Yad. Fiz. 31, 986 (1980) [Sov. J. Nucl. Phys. 31, 509 (1980)].

I. J. Kim, Nucl. Phys. B229, 251 (1983).

3. D. Chen et al., Phys. Rev. Lett. 69, 3286 (1992).

4. E. N. Tsyganov, Fermilab TM-682, TM-684, Batavia, 1976.

5. See, for example, M. Foucher, et al., Phys. Rev. Lett. 68, 3004 (1992).

6. Dong Chen, The Measurement of the Magnetic Moment of Sigma Plus Using Channeling in Bent Crystals, Ph.D thesis (unpublished), State University of New York-Albany, 85 (1992).

7. C. Ankenbrandt et al., Phys. Rev. Lett. 51, 863 (1983).

C. Wilkinson et al., Phys. Rev. Lett. 58, 855 (1987).

8. M. A. Beg, B. W. Lee, and A. Pais, Phys. Rev. Lett. 13, 514 (64). See also J. Franklin, Phys. Rev. 172, 1807 (68).

9. S. Coleman and S. L. Glashow, Phys. Rev. 134, B671 (1964).

10. A. De Rujula, H. Georgi, S. L. Glashow, Phys. Rev. D12, 147 (1975).

11. Ya. I. Azimov, JETP Lett., 53, 554 (1991).

12. A. L. Choudhury and V. Joshi, Phys. Rev. D13, 3115 (1976).

13. D. B. Lichtenberg, Phys. Rev. D15, 345 (1977).

14. P. N. Pandit, et al., Acta Phys. Austriaca 53, 211 (1981).

15. S. N. Jena and D. P. Rath, Phys. Rev. D34, 196 (1986).

16. C. Quigg and J. L. Rosner, Phys. Lett. 71B, 153 (1977), Phys. Rep. 56, 167 (1979).

17. S. K. Bose and L. P. Singh, Phys. Rev. D22, 773 (1980). 
18. Y. Oh, D-P. Min, M. Rho, and N. N. Scoccola, Nucl. Phys. A534, 493 (1991).

19. M. Rho, D. O. Riska, N. N. Scoccola, Phys. Lett. B251, 597 $(1990)$.

20. A. Aleev, et al., Yad. Fiz. 43,619 (1986). (Sov. J. Nucl. Phys. 43, $395(86)$.

21. M. Jezabek, K. Rybicki, and R. Rylko, Phys. Lett. B286, 175 (1992).

22. S. Berman and M. Jacob, Phys. Rev. 139, B1023 (1965).

23. J. Bjorken, Phys. Rev., D40, 1513 (1989).

24. P. Avery, et al., Phys. Rev. Lett., 65, 2842 (1990).

25. H. Albrecht, et al., DESY 91-091 (1991).

26. S. Barlag et al., z. Phys. C49, 555 (1991).

27. J. Appel, Hadroproduction of Charm Particles, Ann. Rev. of Nucl. Sci., 42, 367 (1992).

28. I. J. Kim and C. R. Sun, Proceedings of the Summer study on the Design and Utilization of the SSC (Snowmass), eds $R$. Donaldson and J. Marx (APS, New York), p. 685 (1984).

29. See for example, A. Morelos, et al., Phys. Rev. Lett. 71, 2172 (1993). 
Table 1

TRANSMISSION TABLE

E-761 $\Sigma^{+}$Channeling Magnetic Moment Measurement

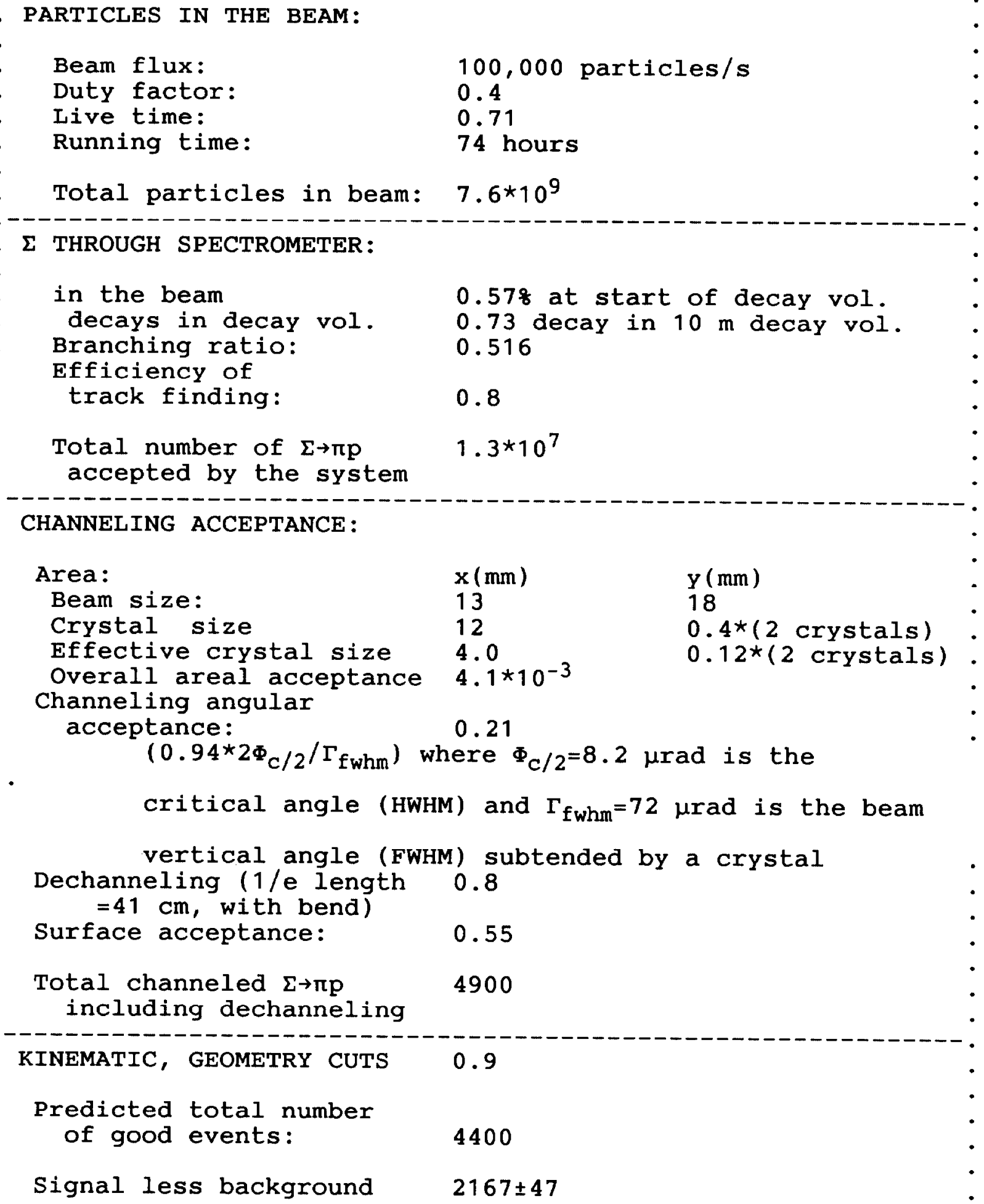

Total particles in beam: $7.6 * 10^{9}$

$\Sigma$ THROUGH SPECTROMETER:

in the beam decays in decay vol.

Branching ratio:

$0.57 \%$ at start of decay vol.

Efficiency of

track finding: $\quad 0.8$

Total number of $\Sigma \rightarrow \pi p \quad 1.3 * 10^{7}$ accepted by the system

0.73 decay in $10 \mathrm{~m}$ decay vol.

0.516

\section{CHANNELING ACCEPTANCE:}

$\begin{array}{llll}\text { Area: } & x(\mathrm{~mm}) & y(\mathrm{~mm}) & \text { : } \\ \text { Beam size: } & 13 & 18 & 0.4 *(2 \text { crystals }) \\ \text { Crystal size } & 12 & 0.12 *(2 \text { crystals }) & \text { : } \\ \text { Effective crystal size } & 4.0 & & \text { : } \\ \text { Overall areal acceptance } & 4.1 * 10^{-3} & & \text {. } \\ \text { Channeling angular } & 0.21 & \end{array}$

$\left(0.94 * 2 \Phi_{\mathrm{c} / 2} / \Gamma_{\mathrm{fwhm}}\right)$ where $\Phi_{\mathrm{c} / 2}=8.2 \mathrm{\mu rad}$ is the

critical angle (HWHM) and $\Gamma_{\mathrm{fwhm}}=72 \mu \mathrm{rad}$ is the beam

vertical angle (FWHM) subtended by a crystal

Dechanneling ( $1 / \mathrm{e}$ length 0.8

$=41 \mathrm{~cm}$, with bend)

Surface acceptance: 0.55

Total channeled $\Sigma \rightarrow \pi p \quad 4900$ including dechanneling

KINEMATIC, GEOMETRY CUTS 0.9

Predicted total number of good events: $\quad 4400$

Signal less background $\quad 2167 \pm 47$ 
Table 2

Possibility of an Improved $\Sigma^{+}$Experiment

\begin{tabular}{|c|c|c|c|}
\hline & $\begin{array}{c}\text { E-761 } \\
\text { Channeling }\end{array}$ & $\begin{array}{l}\text { Improved } \\
\text { Experiment }\end{array}$ & $\begin{array}{l}\text { Ankenbrandt } \\
\text { et al. } \\
\text { PRL 51, } 863 \text { (83) }\end{array}$ \\
\hline $\begin{array}{c}\text { Precession } \\
\text { Angle }\end{array}$ & $60^{\circ}$ & $420^{\circ}$ & $417^{\circ}$ \\
\hline $\begin{array}{l}\text { Vertical } \\
\text { Acceptance }\end{array}$ & $0.12 \mathrm{~mm}$ & $0.5 \mathrm{~mm}$ & \\
\hline $\begin{array}{l}\text { Horizontal } \\
\text { Acceptance }\end{array}$ & $4 \mathrm{~mm}$ & $13 \mathrm{~mm}$ & \\
\hline Crystals & 2 & 4 & \\
\hline Improvement & & 27 & \\
\hline Running Time & 74 hrs & $58 \mathrm{hrs}$ & \\
\hline Events & 2100 & 44,000 & 44,000 \\
\hline
\end{tabular}


Table 3

SOME CHARM BARYON MAGNETIC MOMENT PREDICTIONS: (in nuclear magnetons)

$$
\Lambda_{c^{+}},{\Xi_{c}}^{+}
$$

\begin{tabular}{l|l|}
\hline $\begin{array}{l}\text { Independent Quark Model } \\
\text { ala DGG }\end{array}$ & 0.370 \\
(Pandit et al.) & \\
\hline $\begin{array}{c}\text { Independent Quark Model } \\
\text { log V in spirit of } \\
\text { Quigg-Rosner } \\
\text { (Jena and Rath) }\end{array}$ & 0.352 \\
\hline MIT Bag Model & \\
(Bose and Singh) & 0.503 \\
\hline $\begin{array}{l}\text { Topological Soliton } \\
\text { (Oh, Min, Rho, \& }\end{array}$ & $0.28-$ \\
Scoccola; Riska) & 0.31 \\
\hline
\end{tabular}

